0.74 [0.59-0.94]), absence of radiographic erosions (HR [95\% Cl] 1.52 [1.06$2.20])$ and use of prednisone ( $\mathrm{HR}[95 \% \mathrm{Cl}] 1.51$ [1.36-1.74]). The same predictors were confirmed for point SDAI remission. Irrespective of the criterion, remission was maintained by only half of the patients at follow-up, resulting in sustained remission rates of $28 \%$ for DAS $28 \%$ and $19 \%$ for SDAl in the entire cohort. At multivariate analysis, independent predictors of sustained remission according to either the DAS28 or the SDAI were lower time to achieve remission and deeper remission, whilst demographic factors, baseline disease activity and use of prednisone were not significant (figure 1). Using ROC curve analysis, the best discriminative ability for sustained remission were time to remission $\leq 4$ months, DAS28 at remission $\leq 2.2$ and SDAl at remission $\leq 1$.
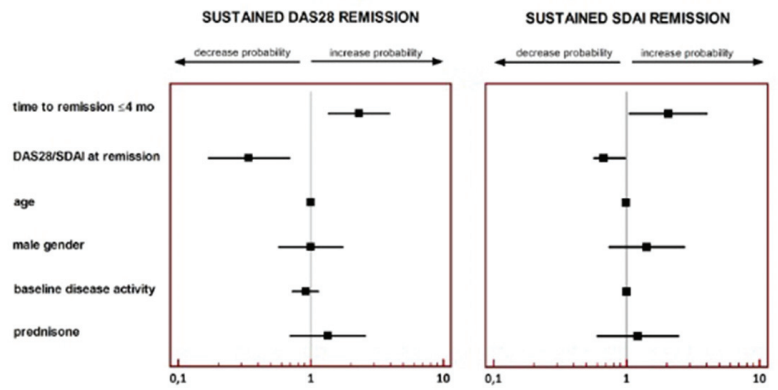

Conclusions: Despite early diagnosis and prompt institution of goal-steered treatment strategies with csDMARDs, only a minority of RA patients experience sustained remission. Remission is more likely to be maintained if the target is attained rapidly after treatment institution and if joint and systemic inflammation are effectively suppressed.

Disclosure of Interest: None declared

DOI: 10.1136/annrheumdis-2018-eular.7548

\section{THU0112 THE INFLUENCE OF AGE AND SEX ON THE PROGRESSION OF RHEUMATOID ARTHRITIS}

I. Gjertsson ${ }^{1}$, J. Nilsson ${ }^{1}$, M. Andersson ${ }^{2}$, M. Leu Agelii ${ }^{1}$, on behalf of BARFOT study group. ${ }^{1}$ Rheumatology and Inflammation Research, University of Gothenburg, Gothenburg; ${ }^{2}$ Clinical Sciences, Section of Rheumatology, Lund University, Lund, Sweden

Background: In Europe, life expectancy is increasing and so does the incidence rheumatoid arthritis (RA), which peaks for both men and women at 70-79 years of age. Further, more than $50 \%$ of patients with RA are $>65$ years of age. To correctly treat and handle all patients with RA irrespective of age there is a need to study the outcome and progression of the disease depending on age of onset as this, to our knowledge, is largely unknown.

Objectives: To study how age at onset of RA influence the course of disease in men and women.

Methods: This study included 2825 patients, $68 \%$ females, from the BARFOT (Better Anti-Rheumatic PharmacOTherapy) early RA cohort. Patients were divided into males and females and into the following age groups: $<40$ years years (yr) $n=415,40-54$ year $n=658,55-69$ year $n=986$ and $\geq 70$ year $n=766$ at onset of disease and inclusion in the study. They were assessed at 3, 6 months and 1, $2,5,8$ years. The following parameters were analysed: DAS28 (disease activity), VAS pain, VAS global health, 28 joint count of tender and swollen joints, respectively, ESR, rheumatoid factor (RF), antibodies to citrullinated proteins (APCA) and Health Assessment Questionnaire (HAQ). The median and 95\% bootstrap confidence interval were calculated in MATLAB R2015a using the bias corrected and accelerated percentile method with 2000 bootstrap samples. Mann-Whitney Utest and Wilcoxon Rank test were used to compare groups, $p<0.01$ was considered as significant due to multiple comparisons.

Results: At inclusion, there were no significant differences in DAS28, VAS global health, VAS pain or tender and swollen joint counts in any of the groups. From 3 months and onward, the DAS28 score were significantly lower for men compared to women in the age groups $<40$ year, 55-69 year and $\geq 70$ year, whereas in the age group 40-54 year there were no significant differences between the sex groups (figure 1). The lowest DAS28 score, at each assessment point, was seen for RF and ACPA positive men $<40$ year and this group had a significantly lower DAS28 score at 8 years compared to all other groups except RF and ACPA negative men and women $\geq 70$ year. At the same time, the worst outcome was seen for RF positive women and for men $\geq 70$ year irrespective of RF, compared to all other groups.
In the age groups $<40$ year, 55-69 year and $\geq 70$ year, both VAS pain and VAS global health were significantly lower in men compared to women from 3-6 month and onward However, no differences were obtained between the sexes in the age group 40-54 year. As expected, HAQ was significantly higher among women than men in all age groups.

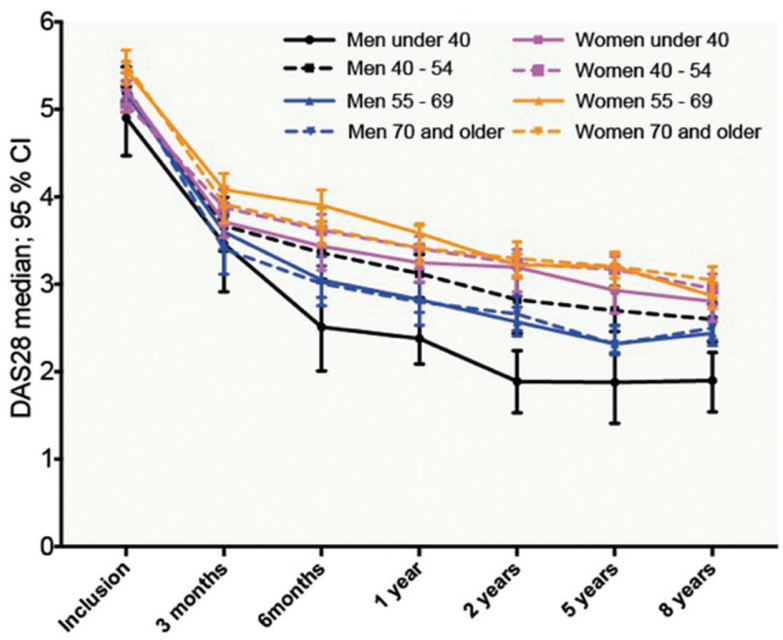

Conclusions: Depending on age at onset, the course of disease, measured as DAS28, differs significantly where seropositive men<40 year have the most favourable prognosis compared to both men and women $\geq 70$ year the worst. No differences in DAS28, VAS global health or pain was found between men and women aged $40-54$ year, which is in contrast to previous studies ${ }^{1}$ comparing men and women without considering the age of onset. The causes for these findings need to be further investigated.

\section{REFERENCE:}

[1] Patients with Early Rheumatoid Arthritis in the 2000s Have Equal Disability and Pain Despite Less Disease Activity Compared with the 1990s: Data from the BARFOT Study over 8 Years. J Rheumatol 2017 Jun;44(6):723731.

Disclosure of Interest: None declared

DOI: 10.1136/annrheumdis-2018-eular.5390

\section{THU0113 LINKING SYSTEMIC ANGIOGENIC MARKERS TO SYNOVIAL VASCULARISATION IN RHEUMATOID ARTHRITIS}

A. Leblond ${ }^{1}$, S. Pezet ${ }^{1}$, A.P. Trouvin ${ }^{2}$, M. Elhai ${ }^{2}$, V. Gonzalez ${ }^{1}$, Y. Allanore ${ }^{2}$, J. Avouac ${ }^{2} .{ }^{1}$ INSERM U1016, Institut Cochin; ${ }^{2}$ Rhumatologie A, Hôpital Cochin, Paris, France

Background: Neoangiogenesis is a crucial event to promote the development of the hyperplasic proliferative pathologic synovium in Rheumatoid arthritis (RA) Ultrasound (US) is sensitive for detection of power Doppler (PD) vascularisation.

Objectives: To explore the associations between a set of complementary circulating angiogenic markers reflecting different angiogenic processes and a com prehensive US assessment in patients with RA.

Methods: Serum levels of eight angiogenic markers (Vascular Endothelia Growth Factor (VEGF), Placenta Growth Factor (PIGF), Tie-2, Angiopoietin-1, soluble Vascular Cell Adhesion Molecule-1 (sVCAM-1), Interleukin-8 (IL-8, CXCL8), CYR61 (CCN1) and Angiostatin), reflecting endothelial cell activation, proliferation, survival, growth and migration, as well as vessel maturation and stabilisation, were measured by quantitative ELISAs in a total of 125 patients with RA, who were all systematically assessed in parallel by PDUS, performed on 32 joints.

Results: Synovitis was detected in 84 patients with RA (67.2\%). Among these patients, 53 patients (42.4\%) had positive Doppler signal, including 31 with moderate to marked hyperemia. Serum levels of soluble Vascular Cell Adhesion Molecule-1 (sVCAM-1) $(808 \pm 293 \mathrm{ng} / \mathrm{mL}$ vs. $697 \pm 240 \mathrm{ng} / \mathrm{mL}, \mathrm{p}=0.022)$ and Tie-2 (16.2 $\pm 7.5 \mathrm{ng} / \mathrm{mL}$ vs. $13.8 \pm 4.9 \mathrm{ng} / \mathrm{mL}, \mathrm{p}=0.038$ ), were more likely to be increased in patients with synovial hyperemia detected on at least one joint (Power Doppler grade $\geq 1$ ). sVCAM-1, Tie-2 and Angiostatin concentrations gradually increased together with the grade of the semiquantitative PDUS scale (figure $1 \mathrm{~A}-\mathrm{C}$ ) and concentrations of these three markers were markedly increased in patients with 